\title{
Computational Statistics and Predictive Analysis in Machine Learning
}

\author{
Deepa H. Kulkarni \\ Assistant Professor, SKN College of Engineering, University of Pune,Maharshtra, India
}

\begin{abstract}
Machine learning evolved from the study of pattern recognition and computational learning theory in intelligence. Machine learning explores the study and construction of algorithms that can learn from and make predictions on data. Such algorithms operate by building a model from example inputs in order to make data-driven predictions or decisions. Machine learning is closely related to and often overlaps with computational statistics; a discipline that also specializes in prediction-making. Machine learning is employed in a range of computing tasks where designing and programming explicit algorithms is infeasible. Example applications include spam filtering, optical character recognition (OCR),search engines and computer vision. Machine learning is sometimes conflated with mining, although that focuses more on exploratory data analysis. Machine learning and pattern recognition "can be viewed as two facets of the same field. When employed in industrial contexts, machine learning methods may be referred to as predictive analytics or predictive analytics
\end{abstract}

Keywords: computational learning, computational statistics, predictive analytics.

\section{Types of Problems and Tasks}

Machine learning tasks are typically classified into three broad categories, depending on the nature of the learning "signal" or "feedback" available to a learning system. These are:

- Supervised learning: The computer is presented with example inputs and their desired outputs, given by a "teacher", and the goal is to learn a general rule that maps inputs to outputs.

- Unsupervised learning: No labels are given to the learning algorithm, leaving it on its own to find structure in its input. Unsupervised learning can be a goal in itself (discovering hidden patterns in data) or a means towards an end.

- Reinforcement learning: A computer program interacts with a dynamic environment in which it must perform a certain goal (such as driving a vehicle), without a teacher explicitly telling it whether it has come close to its goal or not. Another example is learning to play a game by playing against an opponent. ${ }^{[3]: 3}$

Between supervised and unsupervised learning is semisupervised learning, where the teacher gives an incomplete training signal: a training set with some (often many) of the target outputs missing. Transduction is a special case of this principle where the entire set of problem instances is known at learning time, except that part of the targets is missing.

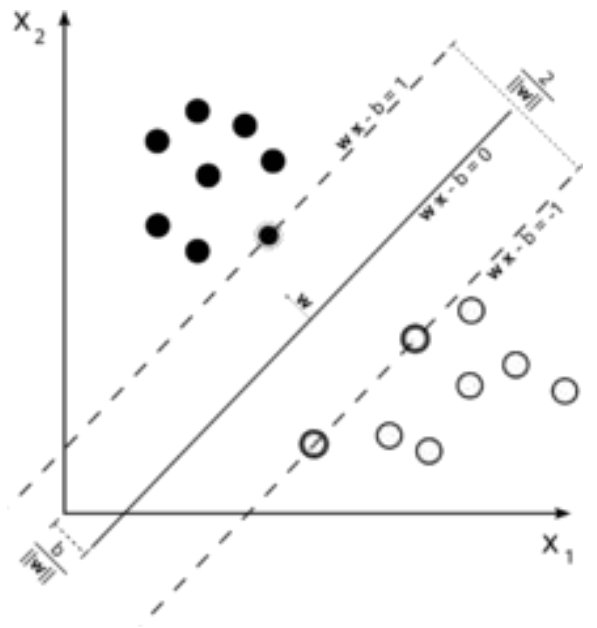

A support vector machine is a classifier that divides its input space into two regions, separated by a linear boundary. Here, it has learned to distinguish black and white circles.

Another categorization of machine learning tasks arises when one considers the desired output of a machine-learned system:

- In classification, inputs are divided into two or more classes, and the learner must produce a model that assigns unseen inputs to one (or multi-label classification) or more of these classes. This is typically tackled in a supervised way. Spam filtering is an example of classification, where the inputs are email (or other) messages and the classes are "spam" and "not spam".

- In regression, also a supervised problem, the outputs are continuous rather than discrete.

- In clustering, a set of inputs is to be divided into groups. Unlike in classification, the groups are not known beforehand, making this typically an unsupervised task.

- Density estimation finds the distribution of inputs in some space.

- Dimensionality reduction simplifies inputs by mapping them into a lower-dimensional space. Topic modeling is a related problem, where a program is given a list of human language documents and is tasked to find out which documents cover similar topics. 


\section{International Journal of Science and Research (IJSR) \\ ISSN (Online): 2319-7064}

Index Copernicus Value (2013): 6.14 | Impact Factor (2014): 5.611

Machine learning, reorganized as a separate field, started to flourish in the 1990s. The field changed its goal from achieving artificial intelligence to tackling solvable problems of a practical nature. It shifted focus away from the symbolic approaches it had inherited from AI, and toward methods and models borrowed from statistics and probability theory. ${ }^{[11]}$ It also benefited from the increasing availability of digitized information, and the possibility to distribute that via the internet.

Machine learning and data mining often employ the same methods and overlap significantly. They can be roughly distinguished as follows:

- Machine learning focuses on prediction, based on known properties learned from the training data.

- Data mining focuses on the discovery of (previously) unknown properties in the data. This is the analysis step of Knowledge Discovery in Databases.

The two areas overlap in many ways: data mining uses many machine learning methods, but often with a slightly different goal in mind. On the other hand, machine learning also employs data mining methods as "unsupervised learning" or as a preprocessing step to improve learner accuracy. Machine learning also has intimate ties to optimization: many learning problems are formulated as minimization of some loss function on a training set of examples. Loss functions express the discrepancy between the predictions of the model being trained and the actual problem instances (for example, in classification, one wants to assign a label to instances, and models are trained to correctly predict the preassigned labels of a set examples). The difference between the two fields arises from the goal of generalization: while optimization algorithms can minimize the loss on a training set, machine learning is concerned with minimizing the loss on unseen samples.

\section{Approaches}

\section{- Decision tree learning}

Decision tree learning uses a decision tree as a predictive model, which maps observations about an item to conclusions about the item's target value.

\section{- Association rule learning}

Association rule learning is a method for discovering interesting relations between variables in large databases.

\section{- Artificial neural networks}

An artificial neural network (ANN) learning algorithm, usually called "neural network" (NN), is a learning algorithm that is inspired by the structure and functional aspects of biological neural networks. Computations are structured in terms of an interconnected group of artificial neurons, processing information using a connectionist approach to computation. Modern neural networks are nonlinear statistical data modeling tools. They are usually used to model complex relationships between inputs and outputs, to find patterns in data, or to capture the statistical structure in an unknown joint probability distribution between observed variables.

\section{- Inductive Logic Programming}

Inductive logic programming (ILP) is an approach to rule learning using logic programming as a uniform representation for input examples, background knowledge, and hypotheses. Given an encoding of the known background knowledge and a set of examples represented as a logical database of facts, an ILP system will derive a hypothesized logic program that entails all positive and no negative examples. Inductive programming is a related field that considers any kind of programming languages for representing hypotheses (and not only logic programming), such as functional programs.

\section{- Support Vector Machines}

Support vector machines (SVMs) are a set of related supervised learning methods used for classification and regression. Given a set of training examples, each marked as belonging to one of two categories, an SVM training algorithm builds a model that predicts whether a new example falls into one category or the other.

\section{- Clustering}

Cluster analysis is the assignment of a set of observations into subsets (called clusters) so that observations within the same cluster are similar according to some predesignated criterion or criteria, while observations drawn from different clusters are dissimilar. Different clustering techniques make different assumptions on the structure of the data, often defined by some similarity metric and evaluated for example by internal compactness (similarity between members of the same cluster) and separation between different clusters. Other methods are based on estimated density and graph connectivity. Clustering is a method of unsupervised learning, and a common technique for statistical data analysis.

\section{- Bayesian Networks}

A Bayesian network, belief network or directed acyclic graphical model is a probabilistic graphical model that represents a set of random variables and their conditional independencies via a directed acyclic graph (DAG). For example, a Bayesian network could represent the probabilistic relationships between diseases and symptoms. Given symptoms, the network can be used to compute the probabilities of the presence of various diseases. Efficient algorithms exist that perform inference and learning.

\section{- Reinforcement Learning}

Reinforcement learning is concerned with how an agent ought to take actions in an environment so as to maximize some notion of long-term reward. Reinforcement learning algorithms attempt to find a policy that maps states of the world to the actions the agent ought to take in those states. Reinforcement learning differs from the supervised learning problem in that correct input/output pairs are never presented, nor sub-optimal actions explicitly corrected.

\section{- Representation Learning}

Several learning algorithms, mostly unsupervised learning algorithms, aim at discovering better representations of the inputs provided during training. Classical examples include principal components analysis and cluster analysis. Representation learning algorithms often attempt to preserve 


\section{International Journal of Science and Research (IJSR) \\ ISSN (Online): 2319-7064}

Index Copernicus Value (2013): 6.14 | Impact Factor (2014): 5.611

the information in their input but transform it in a way that makes it useful, often as a pre-processing step before performing classification or predictions, allowing to reconstruct the inputs coming from the unknown data generating distribution, while not being necessarily faithful for configurations that are implausible under that distribution.

Manifold learning algorithms attempt to do so under the constraint that the learned representation is low-dimensional. Sparse coding algorithms attempt to do so under the constraint that the learned representation is sparse (has many zeros). Multilinear subspace learning algorithms aim to learn low-dimensional representations directly from tensor representations for multidimensional data, without reshaping them into (high-dimensional) vectors. ${ }^{[17]}$ Deep learning algorithms discover multiple levels of representation, or a hierarchy of features, with higher-level, more abstract features defined in terms of (or generating) lower-level features. It has been argued that an intelligent machine is one that learns a representation that disentangles the underlying factors of variation that explain the observed data. $^{[18]}$

\section{- Similarity and Metric Learning}

In this problem, the learning machine is given pairs of examples that are considered similar and pairs of less similar objects. It then needs to learn a similarity function (or a distance metric function) that can predict if new objects are similar. It is sometimes used in Recommendation systems.

\section{- Sparse Dictionary Learning}

In this method, a datum is represented as a linear combination of basis functions, and the coefficients are assumed to be sparse. Let $x$ be a $d$-dimensional datum, $D$ be a $d$ by $n$ matrix, where each column of $D$ represents a basis function. $r$ is the coefficient to represent $x$ using $D$. Mathematically, sparse dictionary learning means the following $x \approx D r$ where $r$ is sparse. Generally speaking, $n$ is assumed to be larger than $d$ to allow the freedom for a sparse representation.

Learning a dictionary along with sparse representations is strongly NP-hard and also difficult to solve approximately. ${ }^{[19]}$ A popular heuristic method for sparse dictionary learning is K-SVD.

Sparse dictionary learning has been applied in several contexts. In classification, the problem is to determine which classes a previously unseen datum belongs to. Suppose a dictionary for each class has already been built. Then a new datum is associated with the class such that it's best sparsely represented by the corresponding dictionary. Sparse dictionary learning has also been applied in image denoising. The key idea is that a clean image patch can be sparsely represented by an image dictionary, but the noise cannot. ${ }^{[20]}$

\section{References}

[1] http:/www.britannica.com/EBchecked/topic/1116194/ machine-learning This is a tertiary source that clearly includes information from other sources but does not name them.

[2] Ron Kohavi; Foster Provost (1998). "Glossary of terms". Machine Learning 30: 271-274.

[3] C. M. Bishop (2006). Pattern Recognition and Machine Learning. Springer. ISBN 0-387-31073-8.

[4] Wernick, Yang, Brankov, Yourganov and Strother, Machine Learning in Medical Imaging, IEEE Signal Processing Magazine, vol. 27, no. 4, July 2010, pp. 2538

[5] Mannila, Heikki (1996). Data mining: machine learning, statistics, and databases. Int'l Conf. Scientific and Statistical Database Management. IEEE Computer Society.

[6] Friedman, Jerome H. (1998). "Data Mining and Statistics: What's the connection?". Computing Science and Statistics 29 (1): 3-9.

[7] Phil Simon (March 18, 2013). Too Big to Ignore: The Business Case for Big Data. Wiley. p. 89. ISBN 978-1118-63817-0.

[8] Mitchell, T. (1997). Machine Learning, McGraw Hill. ISBN 0-07-042807-7, p.2.

[9] Harnad, Stevan (2008), "The Annotation Game: On Turing (1950) on Computing, Machinery, and Intelligence", in Epstein, Robert; Peters, Grace, The Turing Test Sourcebook: Philosophical and Methodological Issues in the Quest for the Thinking Computer, Kluwer

[10] Russell, Stuart; Norvig, Peter (2003) [1995]. Artificial Intelligence: A Modern Approach (2nd ed.). Prentice Hall. ISBN 978-0137903955.

[11] Langley, Pat (2011). "The changing science of machine learning". Machine Learning 82 (3): 275-279. doi:10.1007/s10994-011-5242-y.

[12] Le Roux, Nicolas; Bengio, Yoshua; Fitzgibbon, Andrew (2012). "Improving First and Second-Order Methods by Modeling Uncertainty". In Sra, Suvrit; Nowozin, Sebastian; Wright, Stephen J. Optimization for Machine Learning. MIT Press. p. 404.

[13] MI Jordan (2014-09-10). "statistics and machine learning". reddit. Retrieved 2014-10-01.

[14] Cornell University Library. "Breiman : Statistical Modeling: The Two Cultures (with comments and a rejoinder by the author)". Retrieved 8 August 2015.

[15] Gareth James; Daniela Witten; Trevor Hastie; Robert Tibshirani (2013). An Introduction to Statistical Learning. Springer. p. vii.

[16] Mehryar Mohri, Afshin Rostamizadeh, Ameet Talwalkar (2012) Foundations of Machine Learning, MIT Press ISBN 978-0-262-01825-8.

[17] Lu, Haiping; Plataniotis, K.N.; Venetsanopoulos, A.N. (2011). "A Survey of Multilinear Subspace Learning for Tensor Data" (PDF). Pattern Recognition 44 (7): 1540-1551. doi:10.1016/j.patcog.2011.01.004. edit

[18] Yoshua Bengio (2009). Learning Deep Architectures for AI. Now Publishers Inc. pp. 1-3. ISBN 978-160198-294-0.

[19] A. M. Tillmann, "On the Computational Intractability of Exact and Approximate Dictionary Learning", IEEE Signal Processing Letters 22(1), 2015: 45-49.

[20] Aharon, M, M Elad, and A Bruckstein. 2006. "K-SVD: An Algorithm for Designing Overcomplete 


\section{International Journal of Science and Research (IJSR) \\ ISSN (Online): 2319-7064}

Index Copernicus Value (2013): 6.14 | Impact Factor (2014): 5.611

Dictionaries for Sparse Representation." Signal Processing, IEEE Transactions on 54 (11): 4311-4322

[21] Goldberg, David E.; Holland, John H. (1988). "Genetic algorithms and machine learning". Machine Learning 3 (2): 95-99. doi:10.1007/bf00113892.

[22] Michie, D.; Spiegelhalter, D. J.; Taylor, C. C. (1994). Machine Learning, Neural and Statistical Classification. Ellis Horwood.

[23] Zhang, Jun; Zhan, Zhi-hui; Lin, Ying; Chen, Ni; Gong, Yue-jiao; Zhong, Jing-hui; Chung, Henry S.H.; Li, Yun; Shi, Yu-hui (2011). "Evolutionary Computation Meets Machine Learning: A Survey" (PDF). Computational Intelligence Magazine (IEEE) 6 (4): 68-75. doi:10.1109/mci.2011.942584.

[24] Tesauro, Gerald (March 1995). "Temporal Difference Learning and TD-Gammon". Communications of the ACM 38 (3): 58-68. doi:10.1145/203330.203343.

[25] Daniel Jurafsky and James H. Martin (2009). Speech and Language Processing. Pearson Education. pp. 207 ff.

[26] "BelKor Home Page" research.att.com

[27] "The Netflix Tech Blog: Netflix Recommendations: Beyond the 5 stars (Part 1)". Retrieved 8 August 2015.

[28] Scott Patterson (13 July 2010). "'Artificial Intelligence' Gains Fans Among Investors - WSJ". WSJ. Retrieved 8 August 2015.

[29] When A Machine Learning Algorithm Studied Fine Art Paintings, It Saw Things Art Historians Had Never Noticed, The Physics at ArXiv blog

\section{Author Profile}

Prof. Deepa H. Kulkarni, Assistant Professor, SKN College of Engineering, University of Pune,Maharshtra, India. 